\title{
Cognitive Impairment in Relapsing Remitting and Secondary Progressive Multiple Sclerosis Patients: Efficacy of a Computerized Cognitive Screening Battery
}

\author{
Athanasios Papathanasiou, ${ }^{1,2}$ Lambros Messinis, \\ Vasileios L. Georgiou, ${ }^{3}$ and Panagiotis Papathanasopoulos ${ }^{1}$ \\ ${ }^{1}$ Neuropsychology Section, Department of Neurology, University of Patras Medical School, 26504 Patras, Greece \\ ${ }^{2}$ Department of Neurology, Essex Centre for Neurological Sciences, Queen's Hospital, Romford, RM70AG, UK \\ ${ }^{3}$ Hellenic Open University, 26222 Patras, Greece \\ Correspondence should be addressed to Athanasios Papathanasiou; tpapathanasiou@gmail.com
}

Received 4 January 2014; Accepted 2 February 2014; Published 13 March 2014

Academic Editors: P. Annunziata and Y. Ohyagi

Copyright (C) 2014 Athanasios Papathanasiou et al. This is an open access article distributed under the Creative Commons Attribution License, which permits unrestricted use, distribution, and reproduction in any medium, provided the original work is properly cited.

\begin{abstract}
Objective. To investigate the pattern of cognitive impairment in relapsing remitting multiple sclerosis (RRMS) and secondary progressive multiple sclerosis (SPMS) patients using a computerized battery. Methods. RRMS patients $(N=50)$, SPMS patients $(N=30)$, and controls $(N=31)$ were assessed by Central Nervous System Vital Signs (CNS VS) computerized battery, Trail Making Tests (TMT) A and B, and semantic and phonological verbal fluency tasks. Results. The overall prevalence of cognitive dysfunction was $53.75 \%$ (RRMS 38\%, SPMS 80\%). RRMS patients differed from controls with large effect size on reaction time, medium effect size on TMT A and small on TMT B, phonological verbal fluency, composite memory, psychomotor speed, and cognitive flexibility. SPMS patients differed from controls in all neuropsychological measures (except complex attention) with large effect sizes on TMT A and B, phonological verbal fluency, composite memory, psychomotor speed, reaction time, and cognitive flexibility. Between patient groups, medium effect sizes were present on TMT B and psychomotor speed, while small effect sizes were present on composite memory and processing speed. Conclusion. CNS VS is sensitive in detecting cognitive impairment in RRMS and SPMS patients. Significant impairment in episodic memory, executive function, and processing speed were identified, with gradual increment of the frequency as disease progresses.
\end{abstract}

\section{Introduction}

The functional consequences of cognitive impairment in multiple sclerosis (MS) patients can be devastating. Cognitive impairment has a direct impact on health-related quality of life at all stages of MS [1]. It reduces physical independence and social activities [2], competence in daily activities [3], personal and community independence [4], medication adherence [5], rehabilitation potential [6], and driving safety [7]. Cognitive impaired MS patients are more likely to be unemployed, while employed MS patients are cognitive preserved [8].

Large studies of MS patients have reported cognitive impairment prevalence rates between 40 and $70 \%[9,10]$.
Cognitive impairment has been demonstrated at all stages and in all subtypes of the disease: clinically isolated syndrome (CIS), relapsing remitting multiple sclerosis (RRMS), secondary progressive multiple sclerosis (SPMS), primary progressive multiple sclerosis (PPMS), and even benign multiple sclerosis [11]. However, the more severe levels of cognitive impairment tend to occur in the progressive phase [12]. Although almost all types of cognitive deficits can be observed in MS [13], the typical profile is information processing speed, memory, and executive function impairment, with relative preservation of language [9]. Cognitive status is typically only loosely related to disease duration [14] and physical disability [15], although larger studies have shown significant relations [16]. Cognition can also predict future 
disease progression as cognitive status at CIS stage predicts conversion to MS [17] and cognitive status at MS diagnosis predicts accumulation of physical disability [18].

Traditionally, evaluation of cognitive deficits in MS patients has relied on "paper-and-pencil" neuropsychological batteries. Brief Repeatable Battery of Neuropsychological Tests (BRB-N) and Minimal Assessment of Cognitive Function in MS (MACFIMS) are the most widely used [11]. However, dependence upon neuropsychologists to administer the tests, subjectivity in interpretation, and learning effects that complicate use for follow-up, all undermine their application in widespread clinical practice. In addition, full battery of tests can take several hours to complete, with additional time required for scoring and presentation of results. Computerized cognitive screening batteries have the potential to effectively address these limitations [19].

The aim of this study was to explore the efficacy of Central Nervous System Vital Signs (CNS VS) computerized battery in detecting cognitive dysfunction of MS patients in a district Greek population in Western Greece and try to designate differences in cognitive profile between RRMS and SPMS patients.

\section{Methods}

2.1. Participants. Eighty patients with MS, diagnosed according to McDonald criteria [20], were evaluated in our MS outpatient clinic, University of Patras, Greece. Patients were classified as RRMS $(N=50)$ and SPMS $(N=30)$. Patients with acute relapse during last three months, on corticosteroids or on other medications that could interfere with cognition, known learning disabilities, visual deficits, motor involvement of upper limb, major psychiatric illness, other neurological diseases, and non-Greek origin were excluded from this study. Expanded Disability Status Scale (EDSS) score [21] was also obtained from each patient.

In addition, thirty-one Greek control participants were recruited in order to obtain a sample with similar demographic characteristics to our patients. Exclusion criteria for the control sample included nonnative Greek speakers, visual deficits, learning disabilities, psychiatric or neurological disorder, history of brain injury, cardiovascular illness, medication use that could interfere with cognitive performance, and drug and alcohol consumption.

All groups were assessed with Beck Depression Inventory-Fast Scale in order to exclude major depression as a concomitant factor that could interfere with cognitive performance.

2.2. Neuropsychological Assessment. Neuropsychological assessment was performed with Central Nervous System Vital Signs (CNS VS), a recently developed computerized cognitive screening battery which also provides a Greek adapted version. Brief Core assessment of CNS VS contains seven venerable neuropsychological tests: verbal and visual memory, finger tapping, symbol digit coding, stroop, shifting attention, and continuous performance test. Final results are automatically computed, expressing patient's performance on specific domains such as composite memory, processing speed, psychomotor speed, executive function, reaction time, complex attention, and cognitive flexibility.

In addition, Greek versions of Trail Making Tests A and $\mathrm{B}$, semantic and phonological verbal fluency tasks, and measuring executive function-information processing speed $[22,23]$ were also administered in order to broaden the cognitive screening.

We classified as cognitively impaired, patients who failed on at least $33 \%$ of the included measures [24]. We considered that patients had failed a particular test if they scored 1.5 standard deviation below (or above, if higher score corresponds to worse performance) the average performance of the control group.

2.3. Statistical Analysis. Differences between groups on clinical and demographic characteristics were analyzed using Kruskal-Wallis test for age and education, Mann-Whitney $U$ test for EDSS and duration, and Pearson $\chi^{2}$ test for gender and hand. Pearson correlation coefficients and Spearman rank correlation coefficients were calculated for age, education, EDSS, duration, and neuropsychological measures in all groups. Comparisons of performance between groups in cognitive tasks were investigated using ANOVA and KruskalWallis test (whether or not normality assumption was met by Shapiro-Wilk test). Whenever a statistically significant difference was obtained, pairwise $t$-tests and Mann-Whitney tests using Holm correction were conducted and Hedges' $g$ effect size indicator was calculated.

\section{Results}

3.1. Demographic and Clinical Data. RRMS patients were younger and had lower EDSS scores than SPMS. There were no significant differences in gender distribution and years of education between groups. Disease duration was longer for SPMS group, compared to RRMS (Table 1).

3.2. The Control Group. In the control sample, age and education did not correlate significantly with the performance in neuropsychological tests (Table 2).

3.3. Prevalence of Cognitive Dysfunction. We classified as cognitively impaired patients, those who failed on at least $33 \%$ of the included measures [24]. We considered that patients had failed a particular test if they scored 1.5 standard deviation below (or above, if higher score corresponds to worse performance) the performance of the control group.

The overall prevalence of cognitive dysfunction was $43 / 80$ $=53.75 \%$. Frequency of cognitive dysfunction observed for each group was for RRMS $19 / 50$ patients $(38.00 \%)$ and for SPMS 24/30 patients (80.00\%) (Table 3 ).

3.3.1. Sample of RRMS Patients. Disease duration and EDSS had a weak negative correlation with measures of processing speed, composite memory, and psychomotor speed. Years of education correlated negatively with TMT A, TMT B, reaction time, and complex attention. Age was negatively correlated with performance on semantic and phonological 
TABLE 1: Demographic and clinical characteristics of patients and controls.

\begin{tabular}{lcccc}
\hline & Controls & RRMS & SPMS & Significant differences \\
\hline$N$ & 31 & 50 & 30 & RRMS $<$ SPMS \\
Age (years) & $40.742(2.695)$ & $41.760(11.289)$ & $48.800(6.228)$ & n.s. \\
Education (years) & $12.935(1.093)$ & $12.260(3.556)$ & $12.667(3.262)$ & n.s. \\
Gender M/F, \% & $38.7 / 61.3$ & $22 / 78$ & $26.7 / 73.3$ & RRMS $<$ SPMS \\
EDSS & & $3.050(0.810)$ & $6.183(0.623)$ & RRMS < SPMS \\
Duration (years) & & $8.840(4.053)$ & $15.800(5.135)$ & n.s. \\
Hand, R/L \% & $90.2 / 9.8$ & $94 / 6$ & $93.3 / 6.7$ & \\
\hline
\end{tabular}

Values are mean (SD); $\chi^{2}$ test for gender and hand, Kruskal-Wallis test for age, education, Mann-Whitney $U$ test for EDSS, duration.

RRMS: relapsing remitting multiple sclerosis, SPMS: secondary progressive multiple sclerosis.

n.s: nonsignificant.

Significant differences: RRMS $<$ SPMS with $P<0.001$

TABLE 2: Correlations between age, education, and all neuropsychological measures in our control group. Pearson's correlations for composite memory, executive function, and psychomotor speed. Spearman rank order correlations for all other measures.

\begin{tabular}{|c|c|c|c|c|c|c|c|c|c|c|c|c|}
\hline & Age & Educ. & Tmta & Tmtb & Vfsem & Vfphon & Reactime & Compatt & Cognflex & Procsp & Compm & Psysp \\
\hline Educ. & -0.11 & & & & & & & & & & & \\
\hline Tmta & -0.07 & -0.14 & & & & & & & & & & \\
\hline Tmtb & 0.00 & -0.10 & $0.72^{* * *}$ & & & & & & & & & \\
\hline Vfsem & -0.09 & 0.12 & $-0.44^{*}$ & $-0.59^{* * *}$ & & & & & & & & \\
\hline Vfphon & 0.03 & 0.05 & -0.24 & $-0.42^{* *}$ & $0.69^{* * *}$ & & & & & & & \\
\hline Reactime & 0.14 & -0.23 & 0.16 & 0.01 & 0.13 & -0.03 & & & & & & \\
\hline Compatt & 0.04 & -0.04 & -0.02 & 0.30 & -0.31 & -0.30 & $-0.42^{*}$ & & & & & \\
\hline Cognflex & -0.03 & 0.18 & $-0.47^{* *}$ & $-0.65^{* * *}$ & $0.62^{* * *}$ & $0.49^{* * *}$ & -0.01 & $-0.58^{* * *}$ & & & & \\
\hline Procsp & 0.10 & -0.21 & -0.06 & -0.11 & -0.05 & $-0.37^{*}$ & 0.21 & 0.03 & -0.09 & & & \\
\hline Compm & 0.12 & -0.02 & -0.10 & -0.33 & 0.08 & 0.30 & -0.22 & -0.25 & $0.40^{*}$ & 0.10 & & \\
\hline Psysp & -0.11 & 0.02 & $-0.44^{*}$ & $-0.51^{* *}$ & 0.32 & 0.12 & -0.30 & 0.14 & 0.31 & 0.09 & $0.41^{*}$ & \\
\hline Exfunct & 0.23 & -0.03 & -0.15 & -0.09 & -0.06 & -0.23 & 0.12 & 0.00 & -0.12 & $0.69^{* * *}$ & 0.05 & -0.04 \\
\hline
\end{tabular}

Correlation is significant at $P<0.001^{* * *}, P<0.01^{* *}, P<0.05^{*}$.

Age; Educ.: education; tmta: trail making test a; tmtb: trail making test b; vfsem: verbal fluency semantic; vfphon: verbal fluency phonological; reactime: reaction time; compatt: complex attention; cognflex: cognitive flexibility; procsp: processing speed; compm: composite memory; psysp: psychomotor speed; exfunct: executive function.

verbal fluency, cognitive flexibility, processing speed, composite memory, psychomotor speed, and executive function. On the other hand, disease duration had a positive correlation with reaction time; age was positively correlated with TMT B and reaction time measures, while education had a significant positive correlation with performance on phonological and semantic verbal fluency tasks, processing speed, cognitive flexibility, and executive function (Table 4(a)).

3.3.2. Sample of SPMS Patients. Education had a weak negative correlation with performance on complex attention and weak positive correlation on phonological verbal fluency task. Disease duration was only positively correlated with complex attention, while age and EDSS did not have any significant correlations with task performance (Table 4(b)).

3.4. Comparison of Performance between Groups. Table 5(a) displays performance (mean scores) on all cognitive tasks in RRMS, SPMS patients, and controls.

Since there is a statistically significant difference between mean ages of RRMS and SPMS patients (Table 1), we decided to eliminate its effect by introducing mean age as a covariate when studying the neuropsychological variables in patient groups. To achieve it, we applied a regression analysis on each neuropsychological measure by age and used the residuals of each regression analysis to compare groups on each test.

The statistical comparison (ANOVA, Kruskal-Wallis test) of task performance between RRMS, SPMS, and controls showed statistically significant differences in all measures.

To compare task performance between group pairs, we conducted $t$-test or Mann-Whitney test, whether or not the normality assumption was met by the Saphiro-Wilk normality test. We also calculated Hedges' $g$ as a measure of effect size, which is an unbiased estimator of Cohen's $d$. Particularly, in the normality assumption case, Hedges' $g$ was calculated directly, while in the opposite case, Cliff's delta was calculated and transformed to Cohen's $d$ and then to Hedges' $g$, so that all effect sizes estimate will have the same form and scale. A Hedges' $g$ effect size of 0.2 to 0.3 is considered a "small" effect, around 0.5, a "medium" effect and above 0.8, a "large" effect.

Pairwise comparisons demonstrated that control group performed significantly better than SPMS group in all measures except complex attention. In addition the control group 
TABLE 3: Frequency of impairment in each measure for each group of patients; number and percent $\%$.

\begin{tabular}{lcc}
\hline & RRMS $(N=50)$ & SPMS $(N=30)$ \\
\hline Tmta & $17(34 \%)$ & $19(63.33 \%)$ \\
Tmtb & $17(34 \%)$ & $23(76.67 \%)$ \\
Vfsem & $12(24 \%)$ & $16(53.33 \%)$ \\
Vfphon & $15(30 \%)$ & $15(50 \%)$ \\
Compmem & $8(16 \%)$ & $12(40 \%)$ \\
Psyspeed & $10(20 \%)$ & $20(66.67 \%)$ \\
Reactime & $29(58 \%)$ & $25(83.33 \%)$ \\
Compatt & $12(24 \%)$ & $11(36.76 \%)$ \\
Cognflex & $14(28 \%)$ & $20(66.67 \%)$ \\
Procspeed & $4(8 \%)$ & $8(26.67 \%)$ \\
Exfunction & $12(24 \%)$ & $7(23.33 \%)$ \\
\hline
\end{tabular}

tmta: trail making test a; tmtb: trail making test $b$; vfsem: verbal fluency semantic; vfphon: verbal fluency phonological; compmem: composite memory; psyspeed: psychomotor speed; reactime: reaction time; compatt: complex attention; cognflex: cognitive flexibility; procspeed: processing speed; exfunction: executive function.

RRMS: relapsing remitting multiple sclerosis; SPMS: secondary progressive multiple sclerosis.

performed better than RRMS group in reaction time, TMT A and $\mathrm{B}$, phonological verbal fluency task, composite memory, psychomotor speed, and cognitive flexibility (Table 5(b)).

Large effect sizes were present when SPMS patients were compared to controls on TMT A, TMT B, phonological verbal fluency, composite memory, psychomotor speed, reaction time, and cognitive flexibility. Medium effect sizes were present when the same groups were compared on semantic verbal fluency and executive function, while small effect size was present on processing speed. Comparison between RRMS patients and controls revealed large effect size on reaction time, medium effect size on TMT A and small on TMT B, phonological verbal fluency task, composite memory, psychomotor speed, and cognitive flexibility. Between patient groups (RRMS and SPMS), medium effect sizes were present on TMT B and psychomotor speed, while small effect sizes were present on composite memory and processing speed (Table 5(b)).

\section{Discussion}

The present study allowed the recognition of cognitive impairment in RRMS and SPMS patients in a district Greek population in Western Greece, using CNS VS computerized battery as well as Trail Making Tests A and B and semantic and phonological verbal fluency tasks. The overall prevalence of cognitive dysfunction in our patients was $53.75 \%$, thus in accordance with the estimated prevalence of previous studies that was ranging from $40 \%$ up to $70 \%$ [9]. Cognitive dysfunction declined from RRMS (38\%) to SRMS patients $(80 \%)$, consistent with previous studies suggesting that cognitive deficits are more frequent and pronounced in chronic progressive MS and tend to worsen over time [12].

Higher percentages of impaired RRMS patients were found in TMT A and B (34\%), phonological verbal fluency
TABLE 4: (a) Correlation coefficients between demographic, clinical characteristics, and neuropsychological measures of RRMS patients. Pearson's correlations for composite memory, psychomotor speed, and executive function and Spearman rank order correlations for all other measures. (b) Correlation coefficients between demographic, clinical characteristics, and neuropsychological measures of SPMS patients. Pearson's correlations for composite memory, psychomotor speed, and executive function and Spearman rank order correlations for all other measures.

(a)

\begin{tabular}{lcccc}
\hline & Age & Education & EDSS & Duration \\
\hline Education & $-0.35^{*}$ & & & \\
EDSS & 0.28 & 0.11 & & \\
Duration & $0.58^{* * *}$ & -0.10 & $0.58^{* * *}$ & \\
Tmta & $0.31^{*}$ & $-0.44^{* *}$ & 0.14 & 0.24 \\
Tmtb & $0.43^{* *}$ & $-0.41^{* *}$ & 0.21 & $0.30^{*}$ \\
Vfsem & $-0.44^{* *}$ & $0.42^{* *}$ & -0.09 & -0.20 \\
Vfphon & $-0.41^{* *}$ & $0.46^{* * *}$ & -0.11 & -0.23 \\
Reactime & $0.37^{* *}$ & $-0.32^{*}$ & 0.16 & $0.36^{* *}$ \\
Compatt & 0.23 & $-0.38^{* *}$ & 0.05 & 0.04 \\
Cognflex & $-0.30^{*}$ & $0.40^{* *}$ & -0.13 & -0.19 \\
Procspeed & $-0.60^{* * *}$ & $0.47^{* * *}$ & $-0.31^{*}$ & $-0.43^{* *}$ \\
Compmem & $-0.44^{* *}$ & $0.30^{*}$ & $-0.29^{*}$ & $-0.39^{* *}$ \\
Psyspeed & $-0.44^{* *}$ & $0.33^{*}$ & $-0.40^{* *}$ & $-0.40^{* *}$ \\
Exfunction & $-0.34^{*}$ & $0.44^{* *}$ & -0.13 & -0.20 \\
\hline
\end{tabular}

Correlation is significant at $P<0.001^{* * *}, P<0.01^{* *}, P<0.05^{*}$.

tmta: trail making test a; tmtb: trail making test b; vfsem: verbal fluency semantic; vfphon: verbal fluency phonological; reactime: reaction time; compatt: complex attention; cognflex: cognitive flexibility; procspeed: processing speed; compmem: composite memory; psyspeed: psychomotor speed exfunction: executive function.

(b)

\begin{tabular}{|c|c|c|c|c|}
\hline & Age & Education & EDSS & Duration \\
\hline Education & 0.23 & & & \\
\hline EDSS & 0.07 & 0.05 & & \\
\hline Duration & 0.30 & -0.11 & 0.35 & \\
\hline Tmta & 0.31 & -0.28 & 0.03 & 0.17 \\
\hline Tmtb & 0.00 & -0.17 & -0.11 & -0.07 \\
\hline Vfsem & 0.05 & 0.25 & 0.25 & 0.13 \\
\hline Vfphon & 0.13 & $0.37^{*}$ & 0.10 & 0.09 \\
\hline Reactime & -0.13 & -0.07 & -0.22 & -0.31 \\
\hline Compatt & 0.20 & $-0.43^{*}$ & -0.08 & $0.37^{*}$ \\
\hline Cognflex & 0.00 & 0.10 & 0.15 & -0.12 \\
\hline Procspeed & 0.02 & 0.04 & 0.00 & 0.04 \\
\hline Compmem & 0.10 & 0.07 & -0.32 & 0.14 \\
\hline Psyspeed & -0.32 & 0.17 & -0.28 & -0.26 \\
\hline Exfunction & -0.23 & 0.13 & 0.04 & -0.17 \\
\hline \multicolumn{5}{|c|}{$\begin{array}{l}\text { Correlation is significant at } P<0.001^{* * *}, P<0.01^{* *}, P<0.05^{*} \text {. } \\
\text { tmta: trail making test a; tmtb: trail making test b; vfsem: verbal flu- } \\
\text { ency semantic; vfphon: verbal fluency phonological; reactime: reaction } \\
\text { time; compatt: complex attention; cognflex: cognitive flexibility; procspeed: } \\
\text { processing speed; compmem: composite memory; psyspeed: psychomotor } \\
\text { speed exfunction: executive function. }\end{array}$} \\
\hline
\end{tabular}


TABLE 5: (a) Mean scores (SD) of RRMS, SPMS, and controls on neuropsychological tests. (b) Effect sizes for differences between controls and MS subgroups (RRMS, SPMS). For Cohen's $d$ and Hedges' $g$, an effect size of 0.2 to 0.3 might be a "small" effect, around 0.5 a "medium" effect and 0.8 to infinity, a "large" effect.

(a)

\begin{tabular}{lccc}
\hline & RRMS & SPMS & Controls \\
\hline Tmta & $51.894(19.549)$ & $63.436(16.527)$ & $37.645(12.547)$ \\
Tmtb & $97.543(39.400)$ & $141.537(41.787)$ & $79.646(22.946)$ \\
Vfsem & $43.240(10.413)$ & $36.967(10.950)$ & $47.419(8.865)$ \\
Vfphon & $29.340(12.327)$ & $21.800(9.393)$ & $34.871(9.552)$ \\
Compmem & $90.940(9.851)$ & $83.300(9.675)$ & $97.903(11.915)$ \\
Psyspeed & $135.520(26.301)$ & $103.267(27.875)$ & $148.387(24.204)$ \\
Reactime & $827.360(185.026)$ & $960.433(143.748)$ & $618.516(93.790)$ \\
Compatt & $15.180(10.435)$ & $19.733(9.755)$ & $13.129(6.820)$ \\
Cognflex & $26.400(22.707)$ & $14.200(13.387)$ & $37.968(15.070)$ \\
Procspeed & $39.580(15.617)$ & $26.800(10.387)$ & $40.613(14.986)$ \\
Exfunction & $26.940(22.835)$ & $15.733(13.460)$ & $33.484(16.442)$ \\
\hline
\end{tabular}

RRMS: relapsing-remitting multiple sclerosis; SPMS: secondary progressive multiple sclerosis; tmta: trail making test a; tmtb: trail making test b; vfsem: verbal fluency semantic; vfphon: verbal fluency phonological; compmem: composite memory; psyspeed: psychomotor speed; reactime: reaction time; compatt: complex attention; cognflex: cognitive flexibility; procspeed: processing speed; exfunction: executive function.

(b)

\begin{tabular}{|c|c|c|c|}
\hline & RRMS versus SPMS & RRMS versus Controls & SPMS versus Controls \\
\hline \multirow{2}{*}{ Tmta } & Hedges' $g=0.345$ & Hedges' $g=-0.455^{* *}$ & Hedges' $g=-0.672^{* * *}$ \\
\hline & $P$-value $=0.071$ & $P$-value $=0.001$ & $P$-value $=0.000$ \\
\hline \multirow{2}{*}{ Tmtb } & Hedges' $g=0.770^{* *}$ & Hedges' $g=-0.487^{*}$ & Hedges' $g=-1.313^{* * *}$ \\
\hline & $P$-value $=0.002$ & $P$-value $=0.020$ & $P$-value $=0.000$ \\
\hline \multirow{2}{*}{ Vfsem } & Hedges' $g=-0.280$ & Hedges' $g=0.404$ & Hedges' $g=0.758^{* *}$ \\
\hline & $P$-value $=0.060$ & $P$-value $=0.074$ & $P$-value $=0.002$ \\
\hline \multirow{2}{*}{ Vfphon } & Hedges' $g=-0.391$ & Hedges' $g=0.432^{*}$ & Hedges' $g=0.979^{* * *}$ \\
\hline & $P$-value $=0.085$ & $P$-value $=0.027$ & $P$-value $=0.000$ \\
\hline \multirow{2}{*}{ Compmem } & Hedges' $g=-0.483^{*}$ & Hedges' $g=0.627^{*}$ & Hedges' $g=0.978^{* * *}$ \\
\hline & $P$-value $=0.046$ & $P$-value $=0.013$ & $P$-value $=0.000$ \\
\hline \multirow{2}{*}{ Psyspeed } & Hedges' $g=-0.804^{* *}$ & Hedges' $g=0.455^{*}$ & Hedges' $g=1.226^{* * *}$ \\
\hline & $P$-value $=0.001$ & $P$-value $=0.049$ & $P$-value $=0.000$ \\
\hline \multirow{2}{*}{ Reactime } & Hedges' $g=0.421$ & Hedges' $g=-1.347^{* * *}$ & Hedges' $g=-2.052^{* * *}$ \\
\hline & $P$-value $=0.065$ & $P$-value $=0.000$ & $P$-value $=0.000$ \\
\hline \multirow{2}{*}{ Compatt } & Hedges' $g=0.227$ & Hedges' $g=-0.096$ & Hedges' $g=-0.268$ \\
\hline & $P$-value $=0.319$ & $P$-value $=0.550$ & $P$-value $=0.106$ \\
\hline \multirow{2}{*}{ Cognflex } & Hedges' $g=-0.328$ & Hedges' $g=0.554^{*}$ & Hedges' $g=1.122^{* * *}$ \\
\hline & $P$-value $=0.120$ & $P$-value $=0.010$ & $P$-value $=0.000$ \\
\hline \multirow{2}{*}{ Procspeed } & Hedges' $g=-0.565^{*}$ & Hedges' $g=0.013$ & Hedges' $g=0.513^{*}$ \\
\hline & $P$-value $=0.014$ & $P$-value $=0.957$ & $P$-value $=0.046$ \\
\hline \multirow{2}{*}{ Exfunction } & Hedges' $g=-0.286$ & Hedges' $g=0.287$ & Hedges' $g=0.708^{* *}$ \\
\hline & $P$-value $=0.169$ & $P$-value $=0.187$ & $P$-value $=0.007$ \\
\hline
\end{tabular}

$P<0.001^{* * *}, P<0.01^{* *}, P<0.05^{*}$.

RRMS: relapsing remitting multiple sclerosis; SPMS: secondary progressive multiple sclerosis; CONTR: controls; tmta: trail making test a; tmtb: trail making test b; vfsem: verbal fluency semantic; vfphon: verbal fluency phonological; compmem: composite memory; psyspeed: psychomotor speed; reactime: reaction time; compatt: complex attention; cognflex: cognitive flexibility; procspeed: processing speed; exfunction: executive function.

(30\%), and in reaction time (58\%) with large effect size in reaction time, medium in TMT A (Table 3), and small in TMT B and phonological verbal fluency task (Table 5(b)). We found slightly lower percentages of impaired RRMS patients in cognitive flexibility (28\%), psychomotor speed (20\%) and composite memory (16\%) with significant differences compared to controls and small effect size (Tables 3, 5(b)). The cognitive domains that are mostly affected in our cognitively impaired RRMS patients are processing speed, demonstrated with significantly low reaction time and low scores in TMT 
A, followed by executive dysfunction, showen with poor performance in TMT B and phonological verbal fluency tasks.

SPMS patients performed significantly worse than control group in all tasks except complex attention. Higher percentages of cognitively impaired SPMS patients were found in reaction time $(83.33 \%)$, TMT A and B $(63.33 \%$ and $76.67 \%$ resp.), psychomotor speed and cognitive flexibility $(66.67 \%)$, semantic and phonological verbal fluency tasks (53.33\% and 50\% resp.), and in composite memory (40\%), all with large effect size except from semantic verbal fluency task that had medium effect size (Tables 3, 5(b)).

It appears that deficits in reaction time, TMT A and $\mathrm{B}$, and phonological verbal fluency task are the hallmarks of cognitively impaired RRMS and SPMS patients followed by impaired cognitive flexibility, psychomotor speed, and composite memory. Our findings are consistent with previous studies highlighting that the most affected cognitive domains in MS are processing speed, executive function, and memory with relative preservation of language $[11,25]$.

Comparison between RRMS and SPMS patients demonstrated medium effect sizes on TMT B and psychomotor speed, while small effect sizes were present on composite memory and processing speed (Table 5(b)). Although composite memory, information processing, psychomotor speed, and TMT B deficits are present in RRMS patients, there is a significant decline in performance as disease progress, compatible with previous studies [26, 27].

In the present study, cognitive assessment was performed with CNS VS, a battery that uses computerized forms of traditional tests such as Symbol Digit Modalities and Stroop and can provide even nonneuropsychologist clinician with a reliable, highly sensitive screening tool for detecting cognitive deficits in MS patients. Computerized batteries have demonstrated comparable results to traditional neuropsychological batteries. They are easy to use, require significantly less time to administer, produce instant scoring and can incorporate alternate forms, necessary to minimize learning effect on follow-up. Computerized cognitive screening batteries have also the capacity to accurately-automatically quantify "speed factor" via multiple parameters such as reaction time, psychomotor speed, and processing speed, increasing their sensitivity in detecting even subtle changes in information processing speed [19, 28-33].

However, it is important to address few disadvantages of computerized cognitive assessment screening such as reliance on the visual modality and familiarity of the participant with computers. Moreover, there are many potential sources of error including use of various configurations and operating systems. There is also a provision of less qualitative information compared with paper and pencil tests $[34,35]$.

In order to broaden the neuropsychological assessment and try to designate differences in cognitive profile between RRMS and SPMS patients, we also administered four traditional tests TMT A and B semantic and phonological verbal fluency tasks. A face to face parallel testing by a traditional paper and pencil versus its computerized form will be of great importance to address the actual efficacy of computerized neuropsychological tests in detecting cognitive impairment.
In conclusion, the present study clearly demonstrated cognitive decline in RRMS and SPMS patients of a district Greek population in Western Greece, using a computerized battery (CNS VS) and traditional neuropsychological tests (TMT A and B semantic and phonological verbal fluency tasks). CNS VS appears to be sensitive in detecting cognitive impairment in RRMS and SPMS patients. Significant impairment occurs in almost all studied cognitive domains such as episodic memory, executive function, and processing speed with gradual increment of the frequency as disease progresses. We did not detect distinct patterns of impairment between MS subtypes. This suggests a relatively broad pattern of cognitive deficits in MS, independently of the disease course, but with gradual augmentation of the frequency as disease progresses.

\section{Conflict of Interests}

The authors declare that there is no conflict of interests regarding the publication of this paper.

\section{References}

[1] A. J. Mitchell, J. Benito-León, J. M. González, and J. RiveraNavarro, "Quality of life and its assessment in multiple sclerosis: integrating physical and psychological components of wellbeing," The Lancet Neurology, vol. 4, no. 9, pp. 556-566, 2005.

[2] S. M. Rao, G. J. Leo, L. Ellington, T. Nauertz, L. Bernardin, and F. Unverzagt, "Cognitive dysfunction in multiple sclerosis. II. Impact on employment and social functioning," Neurology, vol. 41, no. 5, pp. 692-696, 1991.

[3] Y. Goverover, H. M. Genova, F. G. Hillary, and J. DeLuca, "The relationship between neuropsychological measures and the timed instrumental activities of daily living task in multiple sclerosis," Multiple Sclerosis, vol. 13, no. 5, pp. 636-644, 2007.

[4] M. P. Amato, G. Ponziani, G. Pracucci, L. Bracco, G. Siracusa, and L. Amaducci, "Cognitive impairment in early-onset multiple sclerosis: pattern, predictors, and impact on everyday life in a 4-year follow-up," Archives of Neurology, vol. 52, no. 2, pp. 168-172, 1995.

[5] J. M. Bruce, L. M. Hancock, P. Arnett, and S. Lynch, "Treatment adherence in multiple sclerosis: association with emotional status, personality, and cognition," Journal of Behavioral Medicine, vol. 33, no. 3, pp. 219-227, 2010.

[6] D. W. Langdon and A. J. Thompson, "Multiple sclerosis: a preliminary study of selected variables affecting rehabilitation outcome," Multiple Sclerosis, vol. 5, no. 2, pp. 94-100, 1999.

[7] T. D. Marcotte, T. J. Rosenthal, E. Roberts et al., "The contribution of cognition and spasticity to driving performance in multiple sclerosis," Archives of Physical Medicine and Rehabilitation, vol. 89, no. 9, pp. 1753-1758, 2008.

[8] K. Honarmand, N. Akbar, N. Kou, and A. Feinstein, "Predicting employment status in multiple sclerosis patients: the utility of the MS functional composite," Journal of Neurology, vol. 258, no. 2, pp. 244-249, 2011.

[9] N. D. Chiaravalloti and J. DeLuca, "Cognitive impairment in multiple sclerosis," The Lancet Neurology, vol. 7, no. 12, pp. 11391151, 2008.

[10] S. M. Rao, G. J. Leo, L. Bernardin, and F. Unverzagt, "Cognitive dysfunction in multiple sclerosis. I. Frequency, patterns, and prediction," Neurology, vol. 41, no. 5, pp. 685-691, 1991. 
[11] D. W. Langdon, "Cognition in multiple sclerosis," Current Opinion in Neurology, vol. 24, no. 3, pp. 244-249, 2011.

[12] D. R. Denney, L. A. Sworowski, and S. G. Lynch, "Cognitive impairment in three subtypes of multiple sclerosis," Archives of Clinical Neuropsychology, vol. 20, no. 8, pp. 967-981, 2005.

[13] R. S. Prakash, E. M. Snook, J. M. Lewis, R. W. Motl, and A. F. Kramer, "Cognitive impairments in relapsing-remitting multiple sclerosis: a meta-analysis," Multiple Sclerosis, vol. 14, no. 9, pp. 1250-1261, 2008.

[14] C. Smestad, L. Sandvik, N. I. Landrø, and E. G. Celius, "Cognitive impairment after three decades of multiple sclerosis," European Journal of Neurology, vol. 17, no. 3, pp. 499-505, 2010.

[15] M. P. Amato, E. Portaccio, B. Goretti et al., "Relevance of cognitive deterioration in early relapsing-remitting MS: a 3-year follow-up study," Multiple Sclerosis, vol. 16, no. 12, pp. 1474-1482, 2010.

[16] S. G. Lynch, B. A. Parmenter, and D. R. Denney, "The association between cognitive impairment and physical disability in multiple sclerosis," Multiple Sclerosis, vol. 11, no. 4, pp. 469-476, 2005.

[17] V. Zipoli, B. Goretti, B. Hakiki et al., "Cognitive impairment predicts conversion to multiple sclerosis in clinically isolated syndromes," Multiple Sclerosis, vol. 16, no. 1, pp. 62-67, 2010.

[18] M. Deloire, A. Ruet, D. Hamel, M. Bonnet, and B. Brochet, "Early cognitive impairment in multiple sclerosis predicts disability outcome several years later," Multiple Sclerosis, vol. 16, no. 5, pp. 581-587, 2010.

[19] A. Achiron, G. M. Doniger, Y. Harel, N. Appleboim-Gavish, M. Lavie, and E. S. Simon, "Prolonged response times characterize cognitive performance in multiple sclerosis," European Journal of Neurology, vol. 14, no. 10, pp. 1102-1108, 2007.

[20] W. I. McDonald, A. Compston, G. Edan et al., "Recommended diagnostic criteria for multiple sclerosis: guidelines from the international panel on the diagnosis of multiple sclerosis," Annals of Neurology, vol. 50, no. 1, pp. 121-127, 2001.

[21] J. F. Kurtzke, "Rating neurologic impairment in multiple sclerosis: an expanded disability status scale (EDSS)," Neurology, vol. 33, no. 11, pp. 1444-1452, 1983.

[22] I. Zalonis, E. Kararizou, N. I. Triantafyllou et al., "A normative study of the trail making test A and B in Greek adults," Clinical Neuropsychologist, vol. 22, no. 5, pp. 842-850, 2007.

[23] M. H. Kosmidis, C. H. Vlahou, P. Panagiotaki, and G. Kiosseoglou, "The verbal fluency task in the Greek population: normative data, and clustering and switching strategies," Journal of the International Neuropsychological Society, vol. 10, no. 2, pp. 164-172, 2004.

[24] M. R. Basso, S. Beason-Hazen, J. Lynn, K. Rammohan, and R. A. Bornstein, "Screening for cognitive dysfunction in multiple sclerosis," Archives of Neurology, vol. 53, no. 10, pp. 980-984, 1996.

[25] L. Messinis, M. H. Kosmidis, E. Lyros, and P. Papathanasopoulos, "Assessment and rehabilitation of cognitive impairment in multiple sclerosis," International Review of Psychiatry, vol. 22, no. 1, pp. 22-34, 2010.

[26] S. C. J. Huijbregts, N. F. Kalkers, L. M. J. de Sonneville, V. de Groot, I. E. W. Reuling, and C. H. Polman, "Differences in cognitive impairment of relapsing remitting, secondary, and primary progressive MS," Neurology, vol. 63, no. 2, pp. 335-339, 2004.

[27] K. K. Zakzanis, "Distinct neurocognitive profiles in multiple sclerosis subtypes," Archives of Clinical Neuropsychology, vol. 15, no. 2, pp. 115-136, 2000.
[28] J. A. Wilken, R. Kane, C. L. Sullivan et al., "The utility of computerized neuropsychological assessment of cognitive dysfunction in patients with relapsing-remitting multiple sclerosis," Multiple Sclerosis, vol. 9, no. 2, pp. 119-127, 2003.

[29] N. Akbar, K. Honarmand, N. Kou, and A. Feinstein, "Validity of a computerized version of the symbol digit modalities test in multiple sclerosis," Journal of Neurology, vol. 258, no. 3, pp. 373-379, 2011.

[30] H. Lapshin, K. L. Lanctot, P. O'Connor, and A. Feinstein, "Assessing the validity of a computer-generated cognitive screening instrument for patients with multiple sclerosis," Multiple Sclerosis, vol. 19, no. 14, pp. 1905-1912, 2013.

[31] A. Ruet, M. S. Deloire, J. Charre-Morin, D. Hamel, and B. Brochet, "A new computerized cognitive test for the detection of information processing speed impairment in multiple sclerosis," Multiple Sclerosis, vol. 19, no. 12, pp. 1665-1672, 2013.

[32] H. Lapshin, B. Audet, and A. Feinstein, "Detecting cognitive dysfunction in a busy multiple sclerosis clinical setting: a computer generated approach," European Journal of Neurology, 2013.

[33] M. R. Piras, I. Magnano, E. D. G. Canu et al., "Longitudinal study of cognitive dysfunction in multiple sclerosis: neuropsychological, neuroradiological, and neurophysiological findings," Journal of Neurology Neurosurgery and Psychiatry, vol. 74, no. 7, pp. 878-885, 2003.

[34] E. Woo, "Computerized neuropsychological assessments," CNS Spectrums, vol. 13, no. 10, supplement 16, pp. 14-17, 2008.

[35] A. N. Cernich, D. M. Brennana, L. M. Barker, and J. Bleiberg, "Sources of error in computerized neuropsychological assessment," Archives of Clinical Neuropsychology, vol. 22, no. 1, pp. 39-48, 2007. 


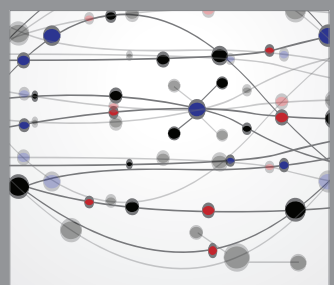

The Scientific World Journal
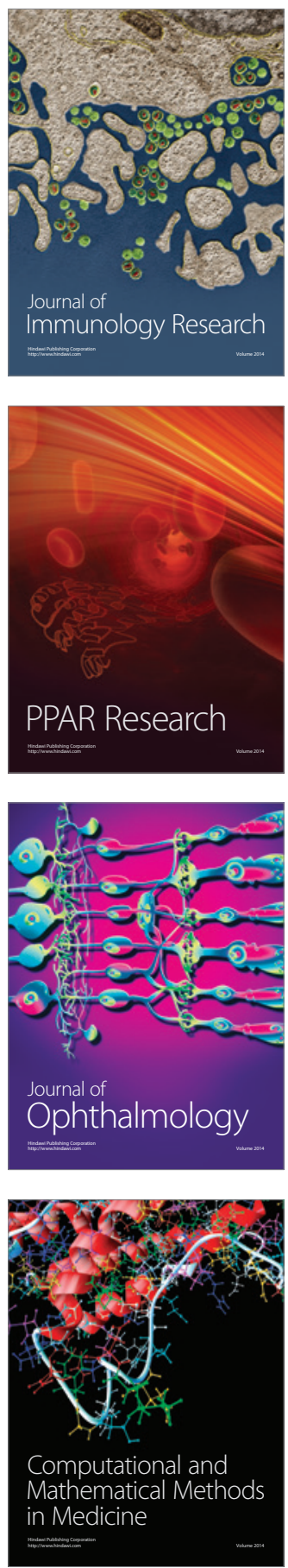

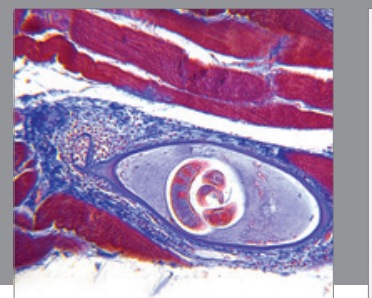

Gastroenterology

Research and Practice
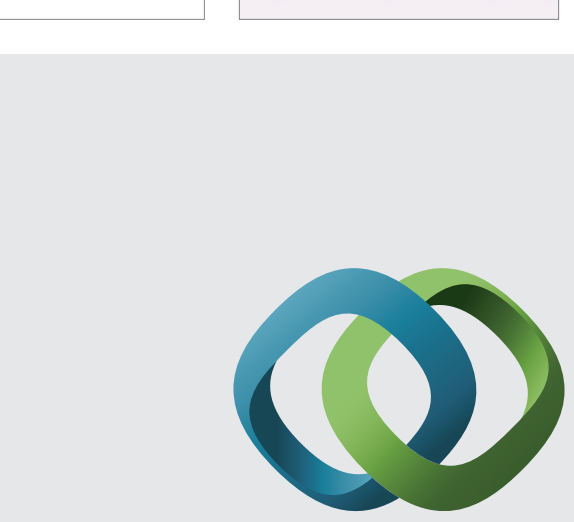

\section{Hindawi}

Submit your manuscripts at

http://www.hindawi.com
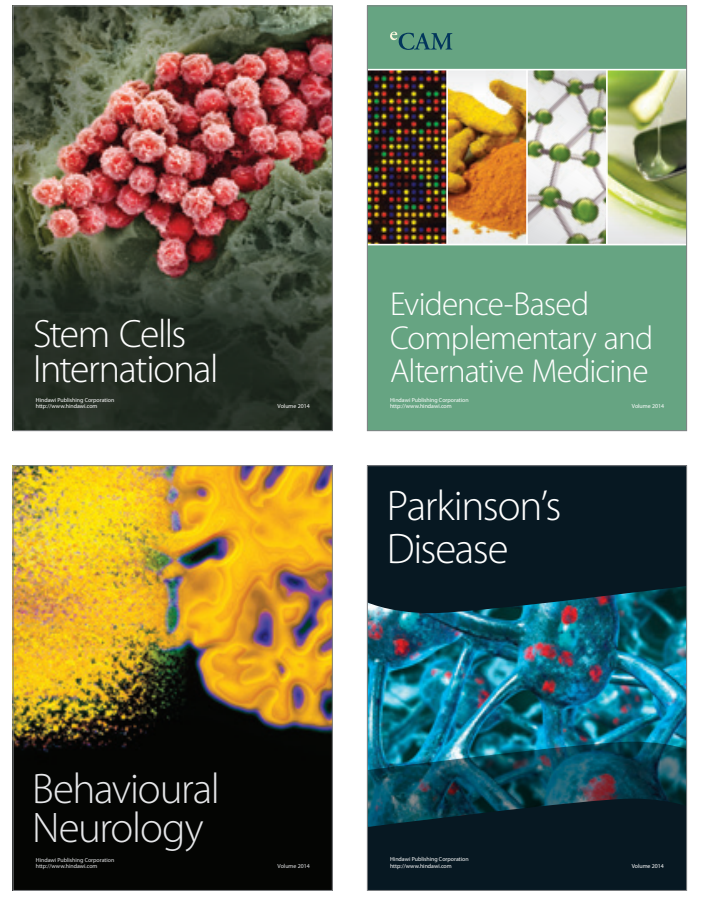
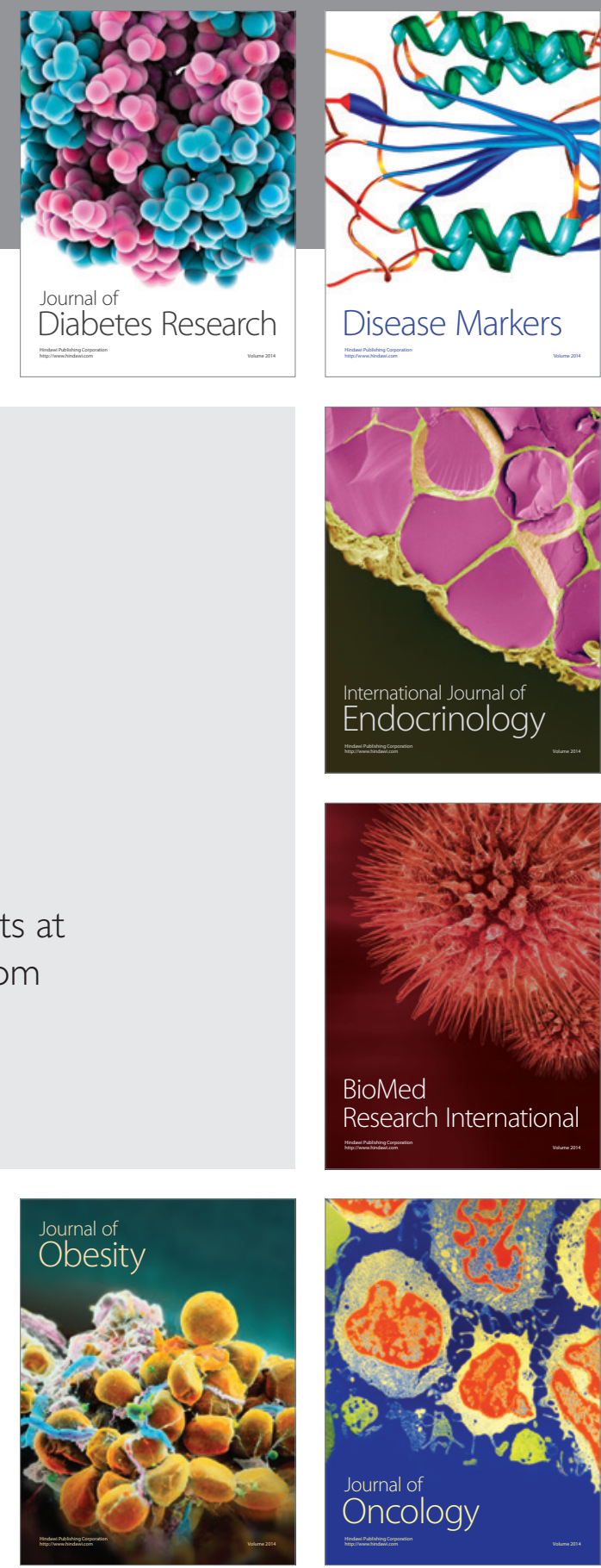

Disease Markers
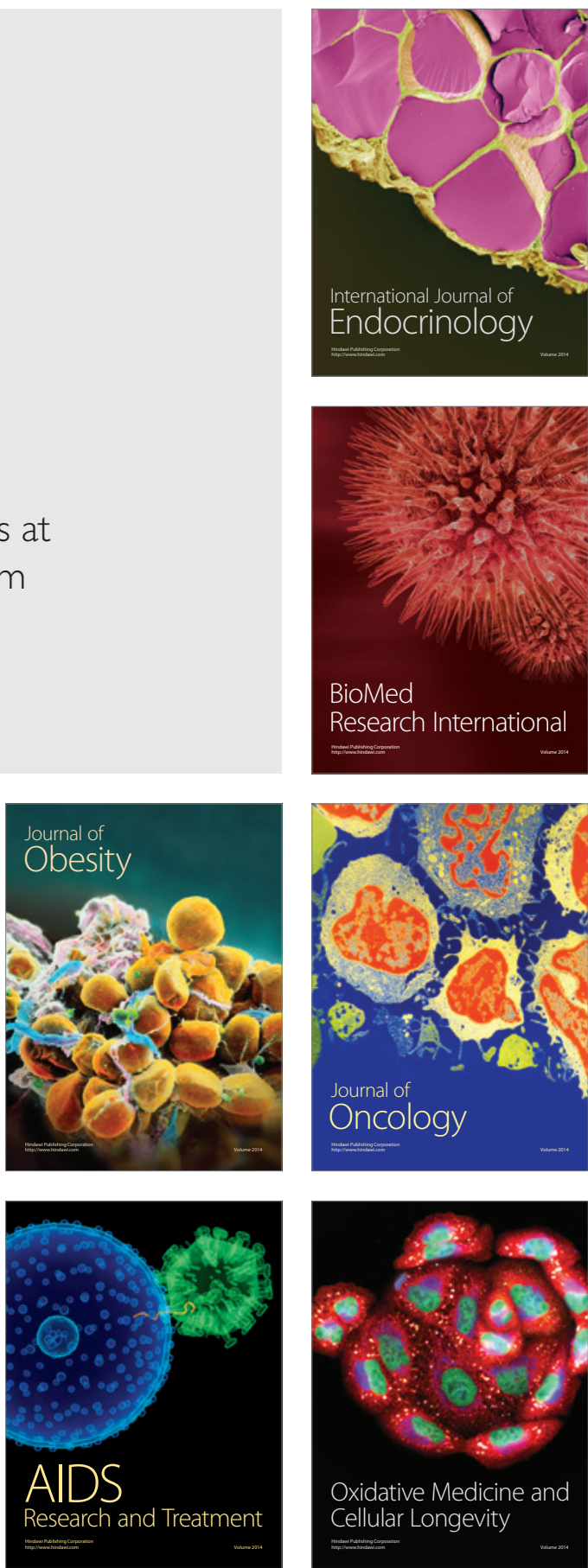